\section{References}

Dean, G. (1949). British Medical Fournal, 1, 842.

Dean, G. (1965). South African Medical Fournal, 39, Suppl., p. 1.

Dean, G. (1967a). British Medical fournal, 2, 724.

Dean, G. (1967b). British Medical Fournal, 4, 420 .

Dean, G. (1967c). South African Medical fournal, 41, 294.
Eadie, J. J., Sutherland, J. M., and Tyrer, J. H. (1965). British Medical Fournal, 1, 1471 .

Kurtzke, J. . F. (1965a). British Medical fournal, 2, 1308.

Kurtzke, J. F. (1965b). Acta Neurologica Scandinavica, 41, 140

Kurtzke, J. F. (1967). British Medical fournal, 3, 738.

Kurtzke, J. F., Dean, G., and Botha, D. P. J. (1970). South African Medical

fournal, 44, 663 .

Poskanzer, D. C., Schapira, K., and Miller, H. (1963). Lancet, 2, 917.

\title{
Idiopathic Parkinsonism Treated with an Extracerebral Decarboxylase Inhibitor in Combination with Levodopa
}

\author{
D. B. CALNE, J. L. REID, S. D. VAKIL, SUMANT RAO, A. PETRIE, C. A. PALLIS, \\ J. GAWLER, P. K. THOMAS, A. HILSON
}

British Medical fournal, 1971, 3, 729-732

\section{Summary}

The clinical actions of levodopa in Parkinsonism, given with and without an extracerebral decarboxylase inhibitor, L-alpha-methyldopahydrazine, were compared. Twenty-one patients were investigated in a "doubleblind cross-over" study, administering levodopa in maximum tolerated dosage. L-Alpha-methyldopahydrazine failed to augment the overall therapeutic actions of levodopa but it consistently alleviated nausea. It is concluded that L-alpha-methyldopahydrazine will prove useful in the management of some Parkinsonian patients who have difficulty in taking levodopa alone.

\section{Introduction}

Accumulating evidence indicates that dopamine is a synaptic transmitter in the brain and that the striatum is severely depleted of dopamine in Parkinsonism. The clinical improvement induced by levodopa is thought to stem from replenishment of dopamine in the central nervous system. Orally administered dopamine is therapeutically inactive because it does not easily cross the blood-brain barrier. Its immediate precursor, levodopa, can, however, enter the brain where decarboxylation to dopamine occurs. Levodopa itself is pharmacologically inert, all its actions being dependent on the production of metabolites.

The therapeutic action of levodopa in Parkinsonism is now well established. Dose-dependent side effects are common, and these may limit its value and sometimes preclude administration. Much of the levodopa given to patients is decarboxylated to catecholamines (dopamine, noradrenaline, and adrenaline) which are in turn converted to phenolic carboxylic acids.

Some of the adverse effects of treatment with levodopa are likely to result from the formation of catecholamines outside

\footnotetext{
Department of Medicine, Royal Postgraduate Medical School, Hammersmith Hospital, London W12 OHS

D. B. CALNE, D.M., M.R.C.P., Lecturer in Neurology

J. L. REID, B.M., M.R.C.P., M.R.C., Research Fellow

S. D. VAKIL, M.B., M.R.C.P., Senior Registrar

SUMANT RAO, M.B., M.R.C.P., Senior Registrar

S. PETRIE, M.SC., Medical Statistician

C. PETRIE, M.SC., Medical Statistician

J. GAWLER, M.B., M.R.C.P., Senior House Officer

Department of Neurology, Royal Free Hospital, London WC1X 8LF P. K. THOMAS, M.D., F.R.C.P., Consultant Neurologist

A. HILSON, M.B., M.R.C.P., Registrar
}

the brain. Bartholini et al. (1967) pointed out that drugs are available which block decarboxylation of levodopa to catecholamines but do not themselves readily cross the blood-brain barrier. Such drugs can be expected to potentiate the therapeutic action of levodopa in several ways. They should reduce those unwanted actions which arise as a result of catecholamine formation at the periphery. By blocking extracerebral metabolism, raised and more sustained plasma levels should be achieved. Initial clinical reports have been encouraging (Barbeau, 1969; Bartholini et al., 1969; Cotzias et al., 1969; Siegfried et al., 1969; Tissot, 1970; Barbeau et al., 1971).

In the following investigation the effects of levodopa were compared with a combination of levodopa plus an extracerebral decarboxylase inhibitor, L-alpha-methyldopahydrazine (Porter et al., 1962). Both regimens were administered in maximum tolerated dosage in a double-blind cross-over study.

\section{Patients and Methods}

Patients. - Twenty-one patients (9 men and 12 women) with idiopathic Parkinsonism were studied. Their ages ranged from 35 to 72 (mean 59.8) years. The criteria for admission to the study were: (1) no evidence of cardiac, hepatic, renal, or haematological disease; (2) availability for outpatient attendance at fortnightly intervals; (3) patient's acceptance of the "blind" protocol for investigation of a new drug. Before treatment 16 of the patients could walk unaided, four could walk only with assistance, and one was unable to walk even with help. They represented a typical group of Parkinsonian patients attending the outpatient department of a general hospital.

Routine Therapy.-Nineteen of the patients had been receiving conventional anti-Parkinsonian drugs (benzhexol, benztropine, orphenadrine) before entering the study. Such treatment was maintained, unaltered, throughout the investigation. None of the patients was taking amantadine, pyridoxine, or monoamine oxidase inhibitors.

Levodopa.-All patients had been receiving tablets of levodopa before admission to the trial. On entering the study they were told that two drug regimens would be tested, but they were not informed that each involved further administration of levodopa. In order to maintain a "blind" setting, the levodopa given during the trial was presented in white capsules of 25,50 , 100 , or $250 \mathrm{mg}$, quite dissimilar in appearance from the $500-\mathrm{mg}$ tablets of levodopa which they had previously received.

L-alpha-methyldopahydrazine.-This extracerebral decarboxylase inhibitor was administered in a dose of $300 \mathrm{mg} /$ day, as capsules of $50 \mathrm{mg}$ which were easily distinguishable from the capsules of levodopa. 
Placebo.-The placebo for L-alpha-methyldopahydrazine was a capsule of identical appearance containing starch.

All the drugs under investigation were given orally in four daily doses taken after food.

\section{DESIGN}

The purpose of the study was to compare the therapeutic and adverse effects of maximum tolerated dosage of levodopa plus a fixed high dose of L-alpha-methyldopahydrazine with the therapeutic and adverse effects of maximum tolerated dosage of levodopa taken without L-alpha-methyldopahydrazine. In the following report levodopa in combination with L-alphamethyldopahydrazine is referred to as regimen $A$ and levodopa without I-alpha-methyldopahydrazine as regimen B. All patients received both regimens of treatment in a "cross-over" design. The first 10 agreeing to take part in the study were allocated to start on regimen $A$ while the remainder began with regimen $B$. This arrangement was determined by factors controlling the supply of drugs, but as there was no selection of the order in which patients presented the design was comparable to one of random allocation.

On entering the trial each patient was admitted to hospital and levodopa tablets were stopped. The first 10 patients, scheduled to start on regimen A, were given $300 \mathrm{mg}$ of L-alpha-methyldopahydrazine per day and this dose was kept constant. They received levodopa capsules in an initial dose related to their previous optimum intake. Those who had been taking under $2 \mathrm{~g}$ of levodopa per day were started on $100 \mathrm{mg} /$ day and the dose was increased by $50 \mathrm{mg} /$ day until side effects were encountered. The dose was then decreased by $50 \mathrm{mg}$ and the patient was discharged from hospital. Those previously receiving more than $2 \mathrm{~g}$ of levodopa per day were started on $200 \mathrm{mg}$ /day, increased by $100 \mathrm{mg} /$ day until unwanted actions appeared, then reduced by $50 \mathrm{mg}$ and discharged. The period of building up the dose of levodopa in hospital lasted 5 to 10 days. The patients returned to the clinic at about fortnightly intervals. Evaluations were performed and the dose of levodopa was adjusted as necessary at each attendance.

Patients were then readmitted for the "cross-over." Regimen A was stopped, and 24 hours later they were given capsules of levodopa without L-alpha-methyldopahydrazine. On this regimen $B$ the starting dose was $0.5 \mathrm{~g}$ of levodopa per day increasing by $250 \mathrm{mg} /$ day for patients who had previously tolerated less than $2 \mathrm{~g} /$ day. Those previously taking over $2 \mathrm{~g} /$ day began with $1 \mathrm{~g} /$ day and increased by $500 \mathrm{mg} /$ day. On reaching maximum tolerated dosage they were discharged from hospital and attended as outpatients fortnightly for a further six weeks.

The last 11 patients to enter the trial were scheduled to start on regimen $\mathbf{B}$. They were admitted to hospital and changed from levodopa tablets to capsules of levodopa which were built up from initially low dosage. This manoeuvre was necessary in order to maintain the "blind" protocol. After a week the patients were discharged and followed up for six weeks as outpatients. They then returned to hospital for the "cross-over." After 24 hours off levodopa they started regimen A. When optimum dosage was reached they were again discharged to attend at fortnightly intervals for six weeks. Eight of the patients who started on regimen $B$ and finished on regimen $A$ were given a placebo instead of L-alpha-methyldopahydrazine at the end of the six-week period of outpatient assessment in order to observe the effect of stopping this drug while maintaining a constant low dose of levodopa.

\section{EVALUATIONS}

The same physician assessed the therapeutic effects of all patients at every attendance. Another physician recorded all the adverse reactions. Neither of these physicians knew the patients' treatment. The first assessment was made immediately after the build-up of dosage had been completed and the remainder at the fortnightly outpatient attendance. After "crossing over" the cycle was repeated on the second regimen.

Therapeutic Actions.-Symptoms (difficulty in dressing, washing, eating, writing) were assessed by questioning the patients and their relatives. Disability was graded from 0 to 4 according to an arbitrarily defined clinical scoring protocol, 0 being normal and 4 representing maximum deficit. Physical signs (tremor, rigidity, speech, facial masking, rising from a chair, balance, finger dexterity, drooling saliva, sweating, gait, posture) were scored in a similar way. Four timed tests were performed-folding a sheet of paper and placing it in an envelope; inserting six pegs into a cribbage board; getting out of a chair, walking six metres, turning, walking back, and sitting down; and writing a standard sentence.

Adverse Reactions. - A standard questionnaire was administered and the answers were arbitrarily graded from 0 (absent) to 4 (severe). Questions were designed to cover known adverse effects of levodopa (anorexia, nausea, vomiting, involuntary movements, giddiness, palpitations, insomnia, euphoria, depression) and problems encountered with routine anti-Parkinsonian drugs (dry mouth, blurred vision, constipation). Experience of hallucinations and delusions was sought, as these can be caused by either levodopa or conventional anti-Parkinsonian therapy. Patients were asked specifically whether they had noticed any rash, because of the possibility that any new drug might provoke a skin eruption. As there had been no previously reported undesirable reactions to $\mathrm{L}$-alpha-methyldopahydrazine, an open question was always presented- "Have you experienced any new problems ?" Finally, blood pressure was recorded with a sphygmomanometer and the pulse by palpation, supine and erect.

\section{Results}

Of the 21 patients 20 completed the full programme of evaluations and investigations. One patient attended for only two assessments on regimen $A$ and was excluded from the study. The first assessment was undertaken in order to acclimatize the patients to the setting in which the evaluations were performed and to introduce them to the examining physicians, the questions, and the timed tests. The results of this assessment are not included in the statistical analysis, and for consistency the initial evaluation after changing drug regimens was also discarded. There remained six assessments for each patient, three on each regimen.

The mean value of the three assessments on each regimen for each patient was determined for each effect and this mean score for each item on regimen A was subtracted from that on regimen $B$. In calculating the estimated treatment effect for each item, expressed as the "benefit" (positive or negative) of regimen $A$ over regimen $B$, allowance was made for the order of administration of the regimens. This allowance was achieved by determining the benefit in the following way. The mean difference between the regimens was calculated for each item over patients for whom regimen A preceded B. The mean difference was similarly determined over patients for whom regimen $B$ preceded $A$. The average of these two means was taken to represent the benefit for each item. The test of significance between the two regimens was thus reduced to testing whether, for each item, this benefit was significantly different from zero. The appropriate test in this instance can be regarded as a modification of the paired $t$ test.

Therapeutic Effect.-Fourteen items were measured to give an overall picture of the patients' clinical status (see material and methods). The estimated treatment benefit for each item is summarized in Table I. From the statistical analysis on these estimated benefits it was evident that there was no significant difference in the therapeutic results obtained on regimens $A$ and 
TABLE I-Estimated "Benefit" of Regimen A over Regimen B on Scores for Individual Therapeutic Effects (Negative Values Represent Deterioration)

\begin{tabular}{|c|c|c|c|c|}
\hline $\begin{array}{l}\text { Therapeutic } \\
\text { Effects }\end{array}$ & - & $\begin{array}{c}\text { Estimated } \\
\text { Benefit } \pm \\
\text { Standard Error }\end{array}$ & $\begin{array}{l}\text { Therapeutic } \\
\text { Bffects }\end{array}$ & $\begin{array}{c}\text { Estimated } \\
\text { Benefit } \pm \\
\text { Standard Error }\end{array}$ \\
\hline 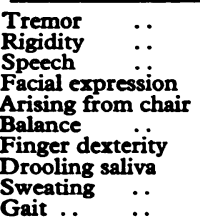 & $\begin{array}{c}\because \\
\because \\
\because \\
\because \\
\because \\
\therefore\end{array}$ & $\begin{array}{l}0.033 \pm 0.078 \\
0.031 \pm 0.093 \\
0.038 \pm 0.129 \\
0.038 \pm 0.091 \\
0.150 \pm 0.095 \\
0.024 \pm 0.062 \\
0.002 \pm 0.168 \\
-0.076 \pm 0.088 \\
-0.037 \pm 0.055 \\
0.074 \pm 0.067\end{array}$ & $\begin{array}{lll}\text { Posture } & . . & . . \\
\text { Dressing } & . & \ldots \\
\text { Washing } & . . & . \\
\text { Eating } & . & . \\
\text { Writing } & . & . \\
\text { Timed envelope test } \\
\text { Timed cribbage test } \\
\text { Timed walking test } \\
\text { Timed writing test }\end{array}$ & 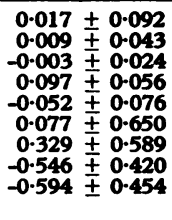 \\
\hline
\end{tabular}

B, when each item was taken alone and also when all 19 items were combined ( $P>0.05)$. Of the eight patients who were given a placebo instead of L-alpha-methyldopahydrazine at the end of the trial while continuing with the same low dose of levodopa which they had been receiving during regimen $\mathbf{A}$ all displayed such striking deterioration that it was considered unethical to extend the observations to other patients.

Adverse Reactions.-The answers to the questionnaire on adverse reactions to treatment were analysed by the technique explained previously and are summarized in Table II. The outstanding difference between the effects of the two regimens was the decreased nausea on regimen $A$ in all 12 patients who

TABLE II-Estimated Benefit of Regimen A over Regimen $B$ on Scores for Individual Adverse Effects (Negative Values Represent Deterioration)

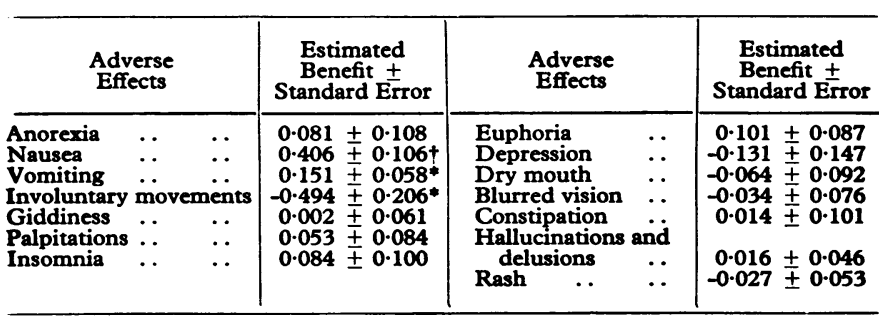

Indicates significance at the $5 \%$ level.

†Indicates significance at the $1 \%$ level.

TABle III-Mean Nausea Scores for Individual Patients. One Patient Starting on Regimen $A$ svas Dropped from the Trial because of Inadequate Attendance, so only 9 Results Appear in this Group

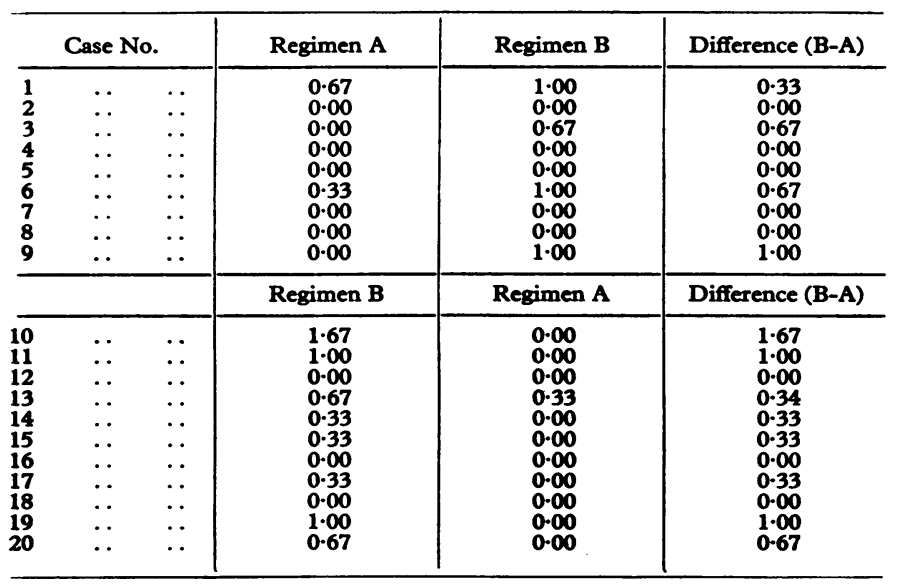

experienced this symptom (Table III). This was significant at the $1 \%$ level. It was also notable that vomiting was reduced on regimen $A$, significant at the $5 \%$ level. In two patients alleviation of nausea was prominent enough for them to be somewhat distressed when L-alpha-methyldopahydrazine was stopped. In these two the advantages conferred by L-alpha-methyldopahydrazine were substantial and would justify continuous treatment on regimen $A$ if the drug were generally available. There was no difference in the gastrointestinal reactions between those patients who started on regimen $A$ and those beginning with regimen $B$, so the improvement achieved by L-alphamethyldopahydrazine cannot be attributed to an artefact arising from tolerance developing to levodopa. The production of new involuntary movements (dyskinesia) was increased on regimen A (significant to the $5 \%$ level). It is probable that this represents an artefact related to patient management rather than a pharmacological reaction (see Discussion). Replies to the open question did not suggest that any important adverse reaction was produced other than those sought in the specific questions.

Cardiovascular Reflexes. - The blood pressure and pulse rate were recorded erect and supine at each attendance. The detailed findings will be reported elsewhere.

Dosages.-The mean maximum tolerated dose of levodopa on regimen A was 0.67 (range 0.1-1.9) g/day and on regimen $B$ 2.97 (range $0 \cdot 8-5.0$ ) $\mathrm{g} /$ day. The degree of potentiation of levodopa by L-alpha-methyldopahydrazine may be represented by expressing the dose of levodopa in regimen $\mathrm{A}$ as a percentage of the dose on regimen $B$. The mean dose of levodopa on regimen $A$ was $22.3 \%$ of that on regimen $B$. There was no correlation between the individual values of this percentage and the maximum tolerated dose of levodopa alone in each patient. The details of doses are summarized in Table IV.

TABLE IV-Mean Maximum Tolerated Dose and Degree of Potentiation of Levodopa by L-alpha-methyldopahydrazine

\begin{tabular}{|c|c|c|c|c|c|c|}
\hline & \multicolumn{3}{|c|}{ Case No. } & \multicolumn{2}{|c|}{$\begin{array}{l}\text { Mean Maximum } \\
\text { Tolerated Dose of } \\
\text { Levodopa (g/day) }\end{array}$} & \multirow{2}{*}{$\begin{array}{c}\text { Degree of Potentiation } \\
\text { (Dose of Levodopa on } \\
\text { Regimen A expressed } \\
\text { as a Percentage of } \\
\text { that on Regimen B) }\end{array}$} \\
\hline & & & & Regimen A & Regimen B & \\
\hline $\begin{array}{r}1 \\
2 \\
3 \\
4 \\
5 \\
6 \\
7 \\
8 \\
9 \\
10 \\
11 \\
12 \\
13 \\
14 \\
15 \\
16 \\
17 \\
18 \\
19 \\
20\end{array}$ & 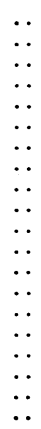 & $\begin{array}{l}\ldots \\
\because \\
\because \\
\because \\
\because \\
\because \\
\because \\
\because \\
\because \\
\because \\
\because \\
\because \\
\end{array}$ & 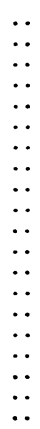 & $\begin{array}{l}0.60 \\
0.10 \\
0.40 \\
0.63 \\
1.87 \\
1.20 \\
0.83 \\
0.17 \\
0.80 \\
1.00 \\
0.30 \\
0.30 \\
0.33 \\
0.45 \\
0.80 \\
0.57 \\
1.10 \\
0.57 \\
0.93 \\
0.47\end{array}$ & $\begin{array}{l}3.58 \\
0.83 \\
2.00 \\
2.67 \\
4.00 \\
4.08 \\
4.00 \\
0.92 \\
5.00 \\
3.75 \\
2.67 \\
2.00 \\
2.25 \\
1.42 \\
3.08 \\
3.75 \\
5.00 \\
3.58 \\
2.00 \\
2.75\end{array}$ & $\begin{array}{l}16 \cdot 8 \% \\
12.1 \% \\
20.0 \% \\
23.6 \% \\
46 \cdot 7 \% \\
29 \cdot 4 \% \\
20.8 \% \\
18.5 \% \\
16 \cdot 0 \% \\
26.7 \% \\
11 \cdot 2 \% \\
15.0 \% \\
14.7 \% \\
31 \cdot 7 \% \\
26.0 \% \\
15.9 \% \\
22.0 \% \\
15.9 \% \\
46.5 \% \\
17 \cdot 1 \%\end{array}$ \\
\hline$\overline{M e}$ & 8 & & & $0.67 \pm 0.09$ & $2.97 \pm 0.27$ & $22 \cdot 3 \pm 2 \cdot 2$ \\
\hline
\end{tabular}

Laboratory Tests.-No important change was detected on regular examination of haemoglobin, white cell count, Coombs test, liver function tests, or blood urea. The serum uric acid was raised in many patients, but this was an artefact due to the technique of analysis which gave spuriously high results in patients receiving levodopa (Cawein and Hewins, 1969).

\section{Discussion}

The administration of L-alpha-methyldopahydrazine to Parkinsonian patients receiving levodopa confers a definite advantage by reducing and often completely abolishing the nausea and vomiting which may be encountered when levodopa is given alone. This antiemetic action has been reported previously (Cotzias et al., 1969). Most patients experience gastrointestinal reactions when starting treatment with levodopa alone, but these symptoms usually regress over a few weeks or months. Sometimes nausea remains and does not respond adequately to conventional antiemetic drugs. In such patients L-alphamethyldopahydrazine may prove very useful. 
The mechanism by which I-alpha-methyldopahydrazine prevents nausea is not known. There is some evidence that levodopa produces gastrointestinal symptoms by increasing the concentration of catecholamines in the brain rather than at the periphery. Thus nausea still occurs when levodopa is given intravenously in man (Degwitz et al., 1960; Fehling, 1960), and vomiting can be produced by giving oral levodopa to dogs who have had their gut denervated surgically (Peng, 1963). Such observations raise the question of whether a peripheral decarboxylase inhibitor could modify an action of levodopa in the central nervous system. In animals the so-called "vomiting centre" is extremely close to the area postrema, a region of the medulla oblongata where the blood-brain barrier is exceptionally permeable and likely to allow entry of L-alpha-methyldopahydrazine. Another possibility is that the emetic action of levodopa is related to the rapid fluctuations in plasma levels which have been recorded (Spiegel et al., 1970; Muenter and Tyce, 1971) and that reduction of nausea is due to the much more evenly sustained plasma concentration of levodopa which is achieved by adding L-alpha-methyldopahydrazine (Dunner et al., 1971).

A rare hazard of administration of levodopa, which was not encountered in our patients, is production of cardiac arrhythmias. This presumably arises from increased extracerebral formation of catecholamines. L-alpha-methyldopahydrazine has been shown to confer protection against arrhythmias induced by levodopa in dogs (Parks et al., 1970), and a similar action can be expected in man. Hence probably a combination of L-alphamethyldopahydrazine with levodopa will emerge as the treatment of choice in Parkinsonian patients with heart disease. As it has been shown that L-alpha-methyldopahydrazine does not block endogenous formation of noradrenaline at the periphery (Henning, 1969) this treatment is unlikely to produce any cardiac complications by sympathetic blockage.

On levodopa alone facial dyskinesia was the commonest doselimiting adverse reaction in our patients. When L-alphamethyldopahydrazine was given in combination with levodopa dyskinesia was the dose-limiting factor in every case. The dyskinesia was more prominent when patients were taking L-alpha-methyldopahydrazine. This is probably attributable to the alleviation of nausea, which allowed the dose of levodopa to be built up at a faster rate than when patients were taking levodopa alone and hence increased the risk of overdosage. As all dyskinesia disappeared on lowering the dose of levodopa, we regard the increased prominence of this side effect when administering L-alpha-methyldopahydrazine as unlikely to prove an important problem.

The maximum tolerated dose of levodopa when given with L-alpha-methyldopahydrazine was reduced to $22 \%$ of the corresponding dose with levodopa alone. Studies currently in progress indicate that, in spite of this disparity in dosage, the maximum plasma levels of levodopa were very similar when patients were on either regimen, which is in accord with our finding that there was no significant difference in the overall therapeutic results achieved on each regimen.

Though we were unable to detect any change in the patients as a whole, it was clearly evident that alleviation of the nausea by L-alpha-methyldopahydrazine in two patients allowed administration of relatively more levodopa: $46 \%$ and $31 \%$ of the maximum tolerated dose of levodopa alone compared with a mean of $22.3 \%$ for the entire group. This difference was statistically significant at the $1 \%$ level. In these two patients there was neurological improvement while L-alpha-methyldopahydrazine was being taken. Sometimes, therefore, this drug is capable of conferring the combined advantages of increased therapeutic effects and reduced adverse reactions. It is speculative to extrapolate from such a limited study, but our results suggest that up to $10 \%$ of patients may derive substantial benefit in this way, and we obtained the impression that more, perhaps up to $30 \%$, may gain worth-while reduction in nausea even if they had not spontaneously complained of this symptom when taking levodopa alone.

Finally, one further advantage can be expected from L-alphamethyldopahydrazine. Pyridoxine blocks the effects of levodopa in Parkinsonism (Duvoisin et al., 1970) and there have been reports of treatment failures attributable to misplaced enthusiasm for multivitamin preparations. Pyridoxine is a codecarboxylase, and by facilitating decarboxylation of levodopa to dopamine through the body the blood levels of levodopa are reduced (Yahr and Duvoisin, 1971). This presumably leads to a decrease in its concentration in the brain. By blocking this codecarboxylase action in extracerebral tissues, L-alphamethyldopahydrazine protects patients receiving levodopa from the deleterious effects of pyridoxine (Cotzias and Papavasiliou, 1971; Yahr and Duvoisin, 1971).

We wish to thank Miss E. Allbutt for continuous help throughout the study. This work was supported by the Wellcome Trust (D.B.C.) and the Medical Research Council (J.L.R.). Merck Sharp \& Dohme Ltd. made a generous contribution towards the administrative costs of the trial and also supplied us with L-alpha-methyldopahydrazine (MK 486) and levodopa. We had considerable help from the pharmaceutical department of Hammersmith Hospital.

\section{References}

Barbeau, A. (1969). Canadian Medical Association fournal, 101, 791. Barbeau, A., Gillo-Joffroy, L., and Mars, H. (1971). Clinical Pharmacology and Therapeutics, 12, 353.

Bartholini, G., Burkard, W. P., Pletscher, A., and Bates, H. M. (1967). Nature, 215, 852

Bartholini, G., Tissot, R., and Pletscher, A. (1969). In Third Symposium on Parkinson's Disease, ed. F. J. Gillingham and I. M. L. Donaldson, p. 192, Edinburgh, Livingstone.

Cawein, M. J., and Hewins, J. (1969). Nero England Fournal of Medicine, 281, 1489.

Cotzias, G. C., and Papavasiliou, P. (1971). Fournal of the American Medical Association, 215, 1504 .

Cotzias, G. C., Papavasiliou, P. S., and Gellene, R. (1969). New England fournal of Medicine, 280, 337.

Degwitz, R., Frowein, R., Kulenkampff, C., and Mohs, V. (1960). Klinische Wochenschrift, 38, 120.

Dunner, D. L., Brodie, H. K. H., and Goodwin, F. K. (1971). Clinical Pharmacology and Therapeutics, 12, 212.

Duvoisin, R. C., Yahr, M. D., and Cote, L. D. (1970). Transactions of the American Neurological Association, $94,81$.

Fehling, C. (1960). Acta Neurologica Scandinavica, 42, 367

Henning, M. (1969). Acta Pharmacologica et Toxicologica, 27, 135.

Muenter, M. D., and Tyce, G. M. (1971). Mayo Clinic Proceedings, 46, 231. Parks, L. C., Watanabe, A. M., and Kopin, I. J. (1970). Lancet, 2, 1014.

Peng M T. (1963). Fournal of Pharmacology and Experimental Therapeutics,

Porter, C. C., Watson, L. S., Titus, D. C., Totars, J. A., and Byer, S. S. (1962). Biochemical Pharmacology, 11, 1067.

Siegfried, W. H., et al. (1969). Pharmacologia Clinica, 2, 23.
Spiegel, H. E., Leon, A. L., and Abrams, W. B. (1970). Personal comegel, H. E.,

Tissot, R. (1970). In L-dopa and Parkinsonism, ed. A. Barbeau and F. H. McDowell, p. 80, Philadelphia, Davis.

Yahr, M. D., and Duvoisin, R. C. (1971). Fournal of the American Medical Association, 216, 2141. 